CORRECTION

https://doi.org/10.1038/s41586-018-0303-z

\title{
Author Correction: Hypothalamic stem cells control ageing speed partly through exosomal miRNAs
}

Yalin Zhang, Min Soo Kim, Baosen Jia, Jingqi Yan,

Juan Pablo Zuniga-Hertz, Cheng Han \& Dongsheng Cai

Correction to: Nature https://doi.org/10.1038/nature23282, published online 26 July 2018.

The microarray data generated and analysed in this Article have been uploaded to the Gene Expression Omnibus (GEO) under accession number GSE113383. The 'Data availability' section of the Methods of the original Article has been corrected. 